\title{
Reflexões sobre o desenvolvimento da Luta Marajoara nas aulas de Educação Física: uma revisão integrativa
}

Reflections on the development of the Luta Marajoara in Physical Education classes: an integrative review

Reflexiones sobre el desarrollo de la Lucha Marajoara en las clases de Educación Física: una revisión integradora

George Almeida Lima

ORCID: https://orcid.org/0000-0003-0899-0427 Universidade Federal do Vale do São Francisco, Brasil E-mail: george_almeida.lima@hotmail.com

Jean Silva Cavalcante

ORCID: https://orcid.org/0000-0002-7084-8408 Universidade Estadual do Ceará, Brasil

E-mail: dr.jeancavalcantefisio@gmail.com

Mabel Dantas Noronha Cisne

ORCID: https://orcid.org/0000-0003-2371-1983

Secretaria Municipal de Educação/Fortaleza, Brasil

E-mail: mabeldantas12@gmail.com

Isabelle Maria Braga da Silva

ORCID: https://orcid.org/0000-0002-4706-8868 Universidade Estadual do Ceará, Brasil E-mail: isabellembragas@gmail.com

Leandro Nascimento Borges

ORCID: https://orcid.org/0000-0001-8592-0436 Centro Universitário Unijaguaribe, Brasil E-mail: leandronborges@gmail.com

Pedro Henrique Silvestre Nogueira

ORCID: https://orcid.org/0000-0002-1533-9387

Centro Universitário Unijaguaribe, Brasil E-mail: pedro.silvestre@fvj.br

Jefferson Florêncio Rozendo

ORCID: https://orcid.org/0000-0002-1723-2049 Instituto Federal de Educação, Ciência e Tecnologia do Estado do Ceará, Brasil

E-mail: jeffersonrozendo@yahoo.com.br

Raphaela Alves Feitosa de Oliveira

ORCID: https://orcid.org/0000-0003-3770-9155 Universidade Federal do Ceará, Brasil E-mail: raphaelaS616@gmail.com

Aline Soares Campos

ORCID: https://orcid.org/0000-0002-2205-4697 Secretaria Estadual de Educação, Brasil E-mail: alinescampos71@gmail.com Heraldo Simões Ferreira

ORCID: https://orcid.org/0000-0003-1999-7982 Universidade Estadual do Ceará, Brasil E-mail: heraldo.simoes@uece.br

\section{Resumo}

Este estudo teve como objetivo discutir sobre o desenvolvimento da Luta Marajoara nas aulas de Educação Física. Este trabalho caracteriza-se como uma revisão integrativa da literatura. O levantamento de dados aconteceu a partir das bases Scielo, Lilacs e Google Scholar, mediante utilização dos descritores: "Luta Marajoara AND Educação Física", "Luta Marajoara AND Educação Básica" e "Luta Marajoara AND Escola". Os resultados apontam que o desenvolvimento desta prática corporal nas aulas de Educação Física é pouco efetivada, pois a formação inicial de professores de Educação Física não apresenta subsídios suficientes para que eles possam se apropriar desta temática de maneira efetiva. Todavia, o desenvolvimento da BNCC apresenta esta prática ao contexto escolar, despertando a curiosidade para a compreensão desta prática corporal. Deste modo, podemos concluir que o professor deve possuir uma formação que propicie a compreensão de metodologias que ampliem a aprendizagem dos alunos sobre os 
aspectos sociais, culturais e motores da Luta Marajoara, facilitando o conhecimento dos alunos sobre esta prática corporal e quebrando estereótipos relacionados ao desenvolvimento das lutas nas aulas de Educação Física.

Palavras-chave: Desenvolvimento; Luta Marajoara; Educação física.

\begin{abstract}
This study aimed to discuss the development of Luta Marajoara in Physical Education classes. This work is characterized as an integrative literature review. Data collection took place from the Scielo, Lilacs and Google Scholar databases, using the descriptors: "Fight Marajoara AND Physical Education", "Fight Marajoara AND Basic Education" and "Fight Marajoara AND School". The results indicate that the development of this corporal practice in Physical Education classes is not very effective, because the initial training of Physical Education teachers does not present sufficient subsidies for them to be able to effectively appropriate this theme. However, the development of the BNCC introduces this practice to the school context, arousing curiosity for the understanding of this corporal practice. In this way, we can conclude that the teacher must provide methodologies that expand students' understanding of the social, cultural and motor aspects of the Marajoara Fight, facilitating students' knowledge of this corporal practice and breaking stereotypes related to the development of fights in Education classes. Physics.
\end{abstract}

Keywords: Development; Marajoara fight; Physical education.

\title{
Resumen
}

Este estudio tuvo como objetivo discutir el desarrollo de Luta Marajoara en las clases de Educación Física. Este trabajo se caracteriza por ser una revisión integrativa de la literatura. La recolección de datos se realizó a partir de las bases de datos Scielo, Lilacs y Google Scholar, utilizando los descriptores: "Fight Marajoara AND Physical Education", "Fight Marajoara AND Basic Education" y "Fight Marajoara AND School". Los resultados muestran que el desarrollo de esta práctica corporal en las clases de Educación Física es poco efectivo, ya que la formación inicial de los profesores de Educación Física no presenta suficientes subsidios para que puedan apropiarse efectivamente de este tema. Sin embargo, el desarrollo de la BNCC introduce esta práctica en el contexto escolar, despertando la curiosidad por la comprensión de esta práctica corporal. De esta forma, podemos concluir que el docente debe brindar metodologías que amplíen la comprensión de los estudiantes sobre los aspectos sociales, culturales y motrices de la Lucha Marajoara, facilitando el conocimiento de los estudiantes sobre esta práctica corporal y rompiendo estereotipos relacionados con el desarrollo de las luchas en la Educación. Clases de Física.

Palabras clave: Desarrollo; Lucha Marajoara; Educación física.

\section{Introdução}

No estado do ParálPA, mais precisamente na Ilha do Marajó, a Luta Marajoara, se configura como uma prática corporal que envolve ações como o agarre, o desequilíbrio, a projeção e o controle do adversário (Antunes; Campos, 2021). Dentro das características apresentadas pela Luta Marajoara, essa prática configura-se como uma luta de curta distância (Espartero, 1999). Segundo Neto et al. (2021, p. 50), esta prática corporal "é praticada pelos nativos da Ilha do Marajó, especialmente dos municípios de Cachoeira do Arari, Soure e Salvaterra, localizados no oeste do Estado do Pará”.

Em concomitância ao aspecto técnico desta prática corporal, está presente o aspecto cultural. Devido à mistura de grupos étnicos distintos, a conflitos e tensões envolvendo estes diversos grupos, emanaram-se transformações culturais na Ilha do Marajó, gerando uma diversidade de crenças, saberes, costumes, práticas sociais e corporais (Pacheco, 2010).

Devido a forte regionalização desta prática, há pouco tempo atrás a Luta Marajoara era transmitida com predominância oral, embasada nas tradições populares da Ilha do Marajó, o que dificultou seu processo de difusão pelas demais regiões do Brasil. Todavia, a Base Nacional Comum Curricular (BNCC), de forma compulsória, inseriu a Luta Marajoara no currículo escolar. Embora essa inclusão facilite sua difusão em âmbito nacional, a BNCC não apresenta referenciais teóricos em que o professor possa se apropriar.

A inserção da Luta Marajoara na unidade temática Lutas na BNCC é compreendida como um avanço para o desenvolvimento de uma visão mais ampla das lutas, tendo em vista que outras regiões do Brasil possuem lutas específicas de seu contexto comunitário e regional, tal como a luta do derruba toco e a Huka-Huka, que são práticas corporais indígenas. Com a homologação do referido documento percebe-se uma preocupação por parte dos pesquisadores na área das lutas, buscando compreender as lutas que são presentes em regiões específicas. 
Podemos perceber que os estudos sobre o desenvolvimento desta prática corporal são recentes, datando o ano de 2018. Todavia, a Luta Marajoara vem sendo objeto de estudos científicos voltados para áreas distintas, como a educação, saúde, cultura e performance (Campos et al., 2018; Santos \& Freitas, 2018; Campos \& Antunes, 2021).

Deste modo, percebemos que a Luta Marajoara está trilhando por dois caminhos específicos. Um no processo educacional, onde ela já está citada na BNCC e outro no processo de esportivização, tendo em vista a criação da Federação Paraense de Luta Marajoara - FPLM. Esses processos podem desencadear o desenvolvimento desta prática no Brasil, todavia, seu processo cultural deve ser valorizado e respeitado (Antunes et al., 2021).

Compreendendo a importância do entendimento da Luta Marajoara como prática cultural, histórica e social, podemos questionar sobre o desenvolvimento desta prática na escola. Deste modo, o presente estudo tem como objetivo discutir sobre o desenvolvimento da Luta Marajoara nas aulas de Educação Física.

\section{Materiais e métodos}

Esse estudo fundamenta-se como uma revisão integrativa de literatura, sintetizando estudos já produzidos, gerando novos resultados, pautados em resultados fundamentados cientificamente (Botelho; Cunha; Macêdo, 2011). A metodologia é de cunho qualitativo e descritivo, objetivando compreender e interpretar as concepções de determinados grupos, buscando analisar os contextos de um fenômeno (Moura, 2021).

As bases de dados utilizadas foram: SCIELO, LILACS e GOOGLE SCHOLAR mediante utilização dos descritores: "Luta Marajoara AND Educação Física", "Luta Marajoara AND Educação Básica" e "Luta Marajoara AND Escola". A utilização das bases de dados justifica-se pela capacidade de agrupar um número significativo de produções acadêmicas. Na análise dos artigos no Google Scholar, percebemos que a partir da segunda página foi percebido um grande distanciamento de artigos que tratavam sobre o objeto desta pesquisa, desta forma, foi adotado como critério: (i) análise da primeira à quarta página, tendo em vista encontrar os melhores artigos. O quadro 1 traz a quantidade de informações encontradas em cada base de dados.

Quadro 1. Quantidade de artigos encontrados nas bases de dados a partir dos descritores.

\begin{tabular}{|c|c|c|c|c|}
\hline TERMOS & SCIELO & LILACS & GOOGLE SCHOLAR & TOTAL \\
\hline $\begin{array}{c}\text { Luta Marajoara } A N D \\
\text { Educação Física }\end{array}$ & 0 & 2 & 40 & $\mathbf{4 2}$ \\
\hline $\begin{array}{c}\text { Luta Marajoara } A N D \\
\text { Educação Básica }\end{array}$ & 0 & 0 & 40 & $\mathbf{4 0}$ \\
\hline $\begin{array}{c}\text { Luta Marajoara } A N D \\
\text { Escola }\end{array}$ & 0 & 0 & 40 & 40 \\
\hline TOTAL & $\mathbf{0}$ & $\mathbf{2}$ & $\mathbf{1 2 0}$ & $\mathbf{1 2 2}$ \\
\hline
\end{tabular}

Fonte: Dados da pesquisa (2021).

Os critérios de inclusão foram: a) obras em português; b) obras que apresentassem uma abordagem que tratasse da Luta Marajoara na Educação Física escolar. Foram critérios para exclusão: a) indisponibilidade completa gratuita em meio eletrônico; b) artigos que não tratavam do objetivo proposto neste estudo. Cabe salientar que a coleta de dados foi iniciada em outubro de 2021 e não houve recorte temporal.

Através dos estudos nas bases de dados foram encontradas um total de 122 obras. Ao realizar a exclusão por duplicidade, foram retirados sete artigos, restando 115 artigos. A partir da leitura e análise do título, foram excluídos 105 
artigos, pois já em seu título apresentavam temáticas diferentes do objetivo deste estudo, permanecendo assim 10 estudos. Após a leitura e análise dos artigos na íntegra e aplicação dos critérios de inclusão e exclusão, foram excluídos sete artigos que apresentavam objetivos diferentes do proposto neste trabalho, restando assim três artigos para análise final. O Fluxograma 1 apresenta o processo de exclusão e inclusão dos artigos.

Fluxograma 1. Processo de inclusão e exclusão de artigos.

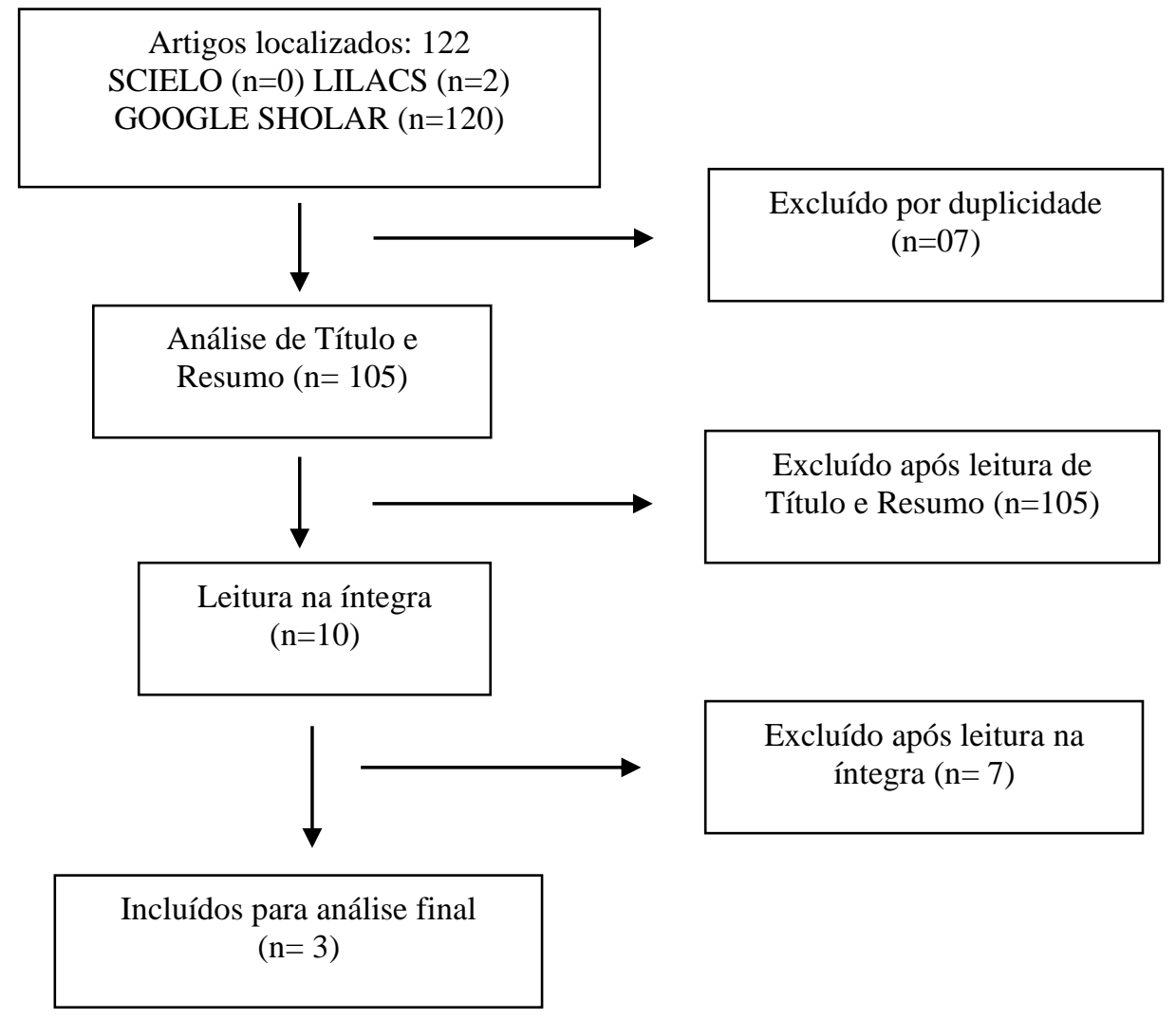

Fonte: Dados da pesquisa (2021).

Os artigos foram analisados a partir da técnica de análise de conteúdo (Bardin, 2011), abrangendo três aspectos: préanálise, que se configura como uma análise para a seleção dos artigos que irão compor a pesquisa; Exploração de material, que consiste na coleta de dados, e a terceira etapa versa sobre o tratamento dos resultados: inferência e interpretação, onde compreende-se a descrição e interpretação dos dados.

\section{Resultados}

A amostra final foi constituída por um total de três publicações, sendo dois artigos de revisão e um estudo de campo (Campos et al., 2018; Santos \& Freitas, 2018; Campos \& Antunes, 2021). O Quadro 2 apresenta dados dos artigos encontrados. 
Quadro 2. Artigos analisados após triagem.

\begin{tabular}{|l|l|l|}
\hline \multicolumn{1}{|c|}{ BASE } & \multicolumn{1}{|c|}{ AUTOR/ANO } & \multicolumn{1}{c|}{ TÍTULO DO ARTIGO } \\
\hline $\begin{array}{l}\text { Revista Brasileira de Ciência e } \\
\text { Movimento. }\end{array}$ & Campos, Pinheiro e Gouveia (2018) & $\begin{array}{l}\text { Modelagem do comportamento técnico da Luta } \\
\text { Marajoara: do desempenho ao educacional. }\end{array}$ \\
\hline $\begin{array}{l}\text { Cadernos de Educação Física e } \\
\text { Esporte. }\end{array}$ & $\begin{array}{l}\text { Santos e Freitas (2018) } \\
\text { educação física escolar em Soure-Marajó. }\end{array}$ \\
\hline Revista Cenas Educacionais. & Campos e Antunes (2021) & $\begin{array}{l}\text { Luta Marajoara: diálogos com o esporte, saúde e } \\
\text { educação. }\end{array}$ \\
\hline
\end{tabular}

Fonte: Dados da pesquisa (2021).

Campos et al. (2018) desenvolveram um estudo que objetivou o desenvolvimento da compreensão de como a Luta Marajoara está sendo desenvolvida a partir da identificação de suas principais técnicas, dos aspectos esportivos e características educativas, buscando encontrar subsídios para sua aplicação no aspecto esportivo e educacional. Como recurso metodológico, os autores realizaram um levantamento na literatura especializada, em fonte documental de treinadores tradicionais e em conversas com atletas renomados da região do Marajó/PA.

A partir dos resultados encontrados, os autores evidenciam que a Luta Marajoara vem se configurando como uma prática que está se expandindo enquanto modalidade de combate. Sua dimensão cultural é um aspecto que vincula esta prática corporal como um conteúdo da Educação Física escolar. Os autores concluem que por mais que a Luta Marajoara possui aspectos relacionados aos esportes de combate, existe a possibilidade de trabalhá-la dentro do contexto da Educação Física escolar, pois esta prática possui caráter sociocultural e educativo, propiciando o desenvolvimento integral dos alunos.

Em estudo proposto por Santos e Freitas (2018) os autores objetivaram discutir sobre a memória e esquecimento sobre os aspectos que envolvem o conhecimento da Luta Marajoara no contexto das aulas de Educação Física no município de Soure-Marajó/PA. Como aspecto metodológico, os autores realizaram uma pesquisa de campo a partir da aplicação de um questionário semiestruturado que envolveu nove professores de Educação Física que atuavam nas escolas do município de Soure-Marajó/PA.

Os resultados encontrados apontam que a maioria dos professores entrevistados não desenvolve o conteúdo da Luta Marajoara em suas aulas. Os autores salientam que aspectos como à memória social, esquecimento histórico e alienação do trabalho são aspectos que dificultam a inserção e a efetivação da Luta Marajoara na Educação Física escolar. Os autores concluem que a Luta Marajoara precisa ser resgatada enquanto aspecto cultural, o que pode contribuir para sua efetivação e valorização nas aulas de Educação Física escolar.

Campos e Antunes (2021) realizaram um estudo que teve como objetivo compreender a Luta Marajoara a partir do pressuposto da interdisciplinaridade, levando em consideração aspectos esportivos, educacionais e sua ligação com a dimensão da saúde. No que concernem os aspectos metodológicos, os autores realizaram um estudo de revisão e de cunho descritivo. As ferramentas de buscas utilizadas foram: Periódicos Capes, Google Acadêmico e obras clássicas.

Os resultados evidenciam que há um avanço no que concerne o reconhecimento sobre a Luta Marajoara enquanto um componente da Educação Física escolar. O desenvolvimento interdisciplinar da Luta Marajoara em consonância com aspectos relacionados ao desenvolvimento da saúde é uma das possibilidades existentes para a efetivação desta temática na escola, para tal, os professores devem buscar recursos metodológicos que incorporem uma percepção do desenvolvimento de hábitos de vida saudáveis e o pensamento crítico sobre as práticas corporais. Os autores concluem que o desenvolvimento da Luta Marajoara na Educação Física escolar não deve acontecer apenas relacionada ao aspecto técnico-esportivo, mas ancorado em pressupostos que possam desenvolver os alunos de maneira integral, viabilizando o trato o desenvolvimento da Luta Marajoara de maneira interdisciplinar. 


\section{Discussões}

A partir da análise dos resultados encontrados, notamos que eles apresentam aspectos relacionados ao desenvolvimento da Luta Marajoara nas aulas de Educação Física. Campos, Pinheiro e Gouveia (2018) e Campos e Antunes (2021) evidenciam que a Luta Marajoara vem se consolidando como um conteúdo da Educação Física escolar, enquanto Santos e Freitas (2018) destacam que há pouca inserção da Luta Marajoara nas aulas de Educação Física e que esta prática precisa ser resgatada enquanto elemento cultural.

A baixa fomentação do conteúdo das lutas na Educação Física escolar, desencadeou o desenvolvimento de pesquisas sobre os motivos para a não efetivação desta prática no currículo escolar (Moura et al., 2017). Alguns motivos são elencados para a não efetivação desta prática, como a ausência de materiais específicos, má formação inicial, falta de apoio das secretarias de Educação e das instituições de ensino, ausência de espaço adequado etc e associação das lutas com a violência (Rufino \& Darido 2012; Matos et al., 2015; Harnisch et al., 2019; Lima, 2021; Lima \& Maia, 2021).

Todavia, algumas ações desencadearam avanços no que concerne a inserção da Luta Marajoara nas aulas de Educação Física, como a elaboração da Base Nacional Comum Curricular (BNCC), que de forma compulsória inseriu as lutas no currículo da educação básica. "[...] além das lutas presentes no contexto comunitário e regional, podem ser tratadas lutas brasileiras (capoeira, huka-huka, luta marajoara etc.) (Brasil, 2017, p. 218, grifo nosso).

Percebemos que a BNCC não apresenta a inserção de apenas uma luta específica. Ela busca abranger o máximo de práticas corporais possíveis, no entanto, há uma menção específica sobre a Luta Marajoara nas aulas de Educação Física. Este fato pode ser considerado um avanço para a efetivação desta prática na escola, pois em contextos anteriores, não existiam documentos norteadores que tratavam especificamente da Luta Marajoara na escola. Os Parâmetros Curriculares Nacionais (PCN's), que ofereciam diretrizes sobre a dinâmica curricular da Educação Física apenas apresentava as lutas dentro da unidade temática esporte.

Moura et al., (2021) evidenciam que a busca pelo convencimento do desenvolvimento das lutas nas aulas de Educação Física parece estar superado, sendo necessário o fomento de debates que possam analisar e apresentar subsídios que proponham aspectos metodológicos para a realização das lutas na escola. Os autores também destacam um aumento nas produções acadêmicas relacionadas a esta área.

Antunes e Campos (2021) destacam que o desenvolvimento da Luta Marajoara está ancorado em dois aspectos: (i) esportivização (ii) escolarização. O processo de esportivização está relacionado com a institucionalização e padronização da modalidade. Todavia, esse processo desencadeia a difusão da modalidade, aproximando a Luta Marajoara dos diversos veículos de comunicação. Cabe salientar que em 2020 foi criada a Federação Paraense de Luta Marajoara (FPLM), ampliando a difusão do viés esportivo.

No que concerne o desenvolvimento da Luta Marajoara a partir do aspecto que envolve a escolarização, Antunes e Campos (2021) destacam que a BNCC acarretou impactos positivos para a efetivação da Luta Marajoara na escola, onde ela é destacada como uma prática regional e como uma luta brasileira, encaixando-se nas unidades temáticas propostas pela BNCC.

Antunes e Campos (2021) evidenciam que o processo de interdisciplinaridade pode favorecer o desenvolvimento da Luta Marajoara nas aulas de Educação Física. Os autores salientam que os professores não devem efetivar seu ensino apenas sobre os aspectos técnicos e gestos motores, mas trazer elementos que ampliem a percepção dos alunos sobre esta prática corporal, como sua associação com a saúde.

Zabala (1998) também destaca que o ensino das práticas corporais não deve estar pautado apenas no aspecto procedimental, que se caracteriza como a realização dos gestos motores, mas também nas dimensões conceituais e atitudinais. Desta forma, o professor deve lançar mão de recursos metodológicos que façam com que os alunos possam entender o 
processo histórico e o desenvolvimento desta prática, bem como quais atitudes os alunos podem ter a partir de sua vivência social.

Campos et al. (2018) enfatizam que a Luta Marajoara está envolvida por aspectos culturais e específicos de uma determinada região do Brasil. Esse fato pode ser explorado de modo a ampliar as possibilidades para o desenvolvimento desta temática nas aulas de Educação Física. Todos os aspectos culturais oriundos desta prática podem ampliar a curiosidade dos alunos, facilitando sua inserção no contexto que envolve a Luta Marajoara.

Santos e Freitas (2018) destacaram que a maioria dos professores que participaram de seu estudo não efetiva a Luta Marajoara nas aulas de Educação Física. Os autores evidenciam que deve haver um resgate cultural para a efetivação desta prática. Todavia, o local de realização da pesquisa foi o município de Soure-Marajó/PA. Podemos perceber que mesmo em escolas do estado do Pará, onde emana forte tradição cultural da Luta Marajoara, ela não é efetivada. Desta forma, percebemos que o resgate cultural possa não ser a única "solução" para a inserção efetiva desta prática na escola.

Outro ponto a ser destacado é a pressão que os professores sofrem para a inserção das práticas corporais mais evidenciadas pelos veículos midiáticos, como o futebol, voleibol, basquetebol e handebol (Rosário \& Darido, 2005). Borges et al., (2021) destacam que existe um distanciamento do debate sobre as lutas na formação de professores de Educação Física, prejudicando a ampliação das percepções sobre esta temática.

Rodrigues e Antunes (2019) destacam que o desenvolvimento das lutas na escola deve estar pautado sobre um planejamento pedagógico bem estruturado que leve em consideração a cultura dos alunos e da prática corporal. Deste modo, o professor precisa lançar mão de recursos pedagógicos que ampliem a concepção dos alunos sobre as diversas práticas corporais existentes. Desta forma, a formação inicial e continuada de professores se configura como uma estratégia eficaz para a ampliação de recursos para o desenvolvimento das lutas na escola (Cisne, et al., 2022).

Seabra, Campos e Antunes (2020) destacam que a inserção da Luta Marajoara no contexto escolar se configura como uma ação complexa, pois envolve uma "nova" prática corporal, que ganhou evidência em todo o currículo da educação básica a partir do fomento da BNCC. Deste modo, os autores salientam que a formação de professores é um aspecto essencial para a ampliação do desenvolvimento desta prática.

Podemos perceber que as discussões sobre o desenvolvimento da Luta Marajoara nas aulas de Educação Física estão em fase inicial, datando o ano de 2018. Este aspecto pode ser compreendido pelo fato de a Luta Marajoara ser citada na BNCC em 2017. Antes da busca pela diversificação dos conteúdos da Educação Física, seu processo de ensino estava centrado nos esportes coletivos mais evidenciados pelos veículos midiáticos, como o futsal/futebol, basquete, handebol e voleibol (Darido \& Rangel, 2014).

\section{Considerações Finais}

O objetivo deste estudo foi discutir sobre o desenvolvimento da Luta Marajoara nas aulas de Educação Física escolar. Com base na pesquisa e nos dados analisados, podemos destacar que esta modalidade ainda é pouco efetivada na Educação Física escolar, apesar das discussões sobre seu resgate cultural e efetivação nas aulas de Educação Física estarem em seu início.

Compreendemos que a BNCC pode ser considerada um documento incentivador à inclusão de novas práticas corporais, de forma específica com a temática de lutas brasileiras, onde a Luta Marajoara está inserida. Passo esse importante e decisivo para o desenvolvimento da modalidade nas aulas de Educação Física. Entretanto, o fato de esta prática estar relacionada culturalmente a uma região específica do país não parece ser suficiente para sua inserção efetiva na escola, sendo necessária a ampliação de debates que tratem do desenvolvimento desta temática nas aulas de Educação Física escolar. 
Diversos estudos têm apontado à importância da efetivação da prática da Luta Marajoara nas aulas de Educação Física, aproximando relações entre esta prática corporal e as instituições de ensino. Desta forma, as discussões sobre o desenvolvimento da Luta Marajoara como elemento de ensino da Educação Física escolar podem se tornar cada vez mais consistentes, dando suporte aos professores para que possam continuar com a difusão desta modalidade.

Deste modo, este estudo não teve como objetivo encerrar as discussões sobre a temática em questão, mas apontar subsídios para seu melhor desenvolvimento na Educação Física escolar. Deste modo, fazem-se necessárias novas investigações sobre os aspectos metodológicos de ensino da Luta Marajoara.

\section{Referências}

Antunes, M. M. \& Campos, I. S. L. (2021). Luta Marajoara: aspectos técnicos, esportivos e pedagógicos. In: Antunes, M. M. \& Moura, D. L (Orgs). Dialogando com as lutas, artes marciais e esportes de combate. Curitiba. Editora CRV.

Antunes, M. M., Borba-Pinheiro, C. J., \& Campos, Í. L. (2021). Luta marajoara: uma luta genuinamente brasileira. Curitiba, PR: Revista ProAtiva. V.5, E5024 .

Bardin, L. Análise de conteúdo. (2011). Edições 70.

Botelho, L. L. R., de Almeida Cunha, C. C., \& Macedo, M. (2011). O método da revisão integrativa nos estudos organizacionais. Gestão e sociedade, 5(11), 121-136.

Borges, L. N., Fernandes, M. P. R., Cisne, M. D. N., \& Ferreira, H. S. (2021). Formação de professores para o ensino de lutas na educação física escolar: o estado da questão. Revista Ibero-Americana de Estudos em Educação, 1547-1561.

Brasil. (2017) Ministério da Educação. Base Nacional Comum Curricular. MEC.

Campos, Í. S. L., Pinheiro, C. J., \& Gouveia, A. (2019). Modelagem do comportamento técnico da Luta Marajoara: do desempenho ao educacional. R. bras. Ci. e Mov 2019; 27 (2): 209, 217.

Campos, I. S. L., \& Antunes, M. M. (2021). Luta Marajoara: diálogos com o esporte, saúde e educação. Cenas Educacionais, 4, e11870-e11870.

Cisne, M. D. N., Fernandes, M. P. R., Borges, L. N., Barroso, M. L., Nogueira, P. H. S., Almeida, M. I. M., \& Ferreira, H. S. (2022). Formação e prática pedagógica na Educação Física escolar: a percepção dos professores sobre a temática lutas. Research, Society and Development, 11(1), e43511125212e43511125212.

Darido, S. C. \& Rangel, I. C. (2014). Educação Física na Escola: implicações para a prática pedagógica. Guanabara Koogan.

Espartero, J. (1999). Aproximación histórico-conceptual a los deportes de lucha. In: Villamón, M. Introducción al Judo. Barcelona: Editorial hispano Europea S.A.

Harnisch, G. S., Walter, L. W., de Oliveira Guilherme, S. M., Silva, B. P., Lottermann, A. L. F., \& Borella, D. R. (2018). As lutas na educação física escolar: um ensaio sobre os desafios para sua inserção. Caderno de Educação Física e Esporte, 16(1), 179-184.

Lima, G. A. (2021). Ensino das lutas na escola: um estudo com professores de educação física da cidade de Campos Sales-ce. Temas em Educação Física Escolar, 6(1), 71-86.

Lima, G. A., \& da Silva Maia, F. E. (2021). Os impactos da arte marcial no comportamento dos seus praticantes. Revista Interfaces: Saúde, Humanas e Tecnologia, 9(2), 1098-1104.

Matos, J. A. B., Hirama, L. K., Galatti, L. R., \& Montagner, P. C. (2015). A presença/ausência do conteúdo lutas na educação física escolar: identificando desafios e propondo sugestões. Conexões, 13(2), 117-135.

Moura, D. L. et al., Dialogando sobre o ensino da Educação Física: lutas na escola. CRV, 2017.

Moura, D. L. et al., As lutas na Educação Física escolar: uma análise sobre a BNCC. (2021). In: Antunes, M. M.; Moura, D. L (Orgs). Dialogando com as lutas, artes marciais e esportes de combate. Editora CRV.

Moura, D. L. (2021). Pesquisa Qualitativa: um guia prático para pesquisadores iniciantes. CRV.

Neto, R. F. E; Abrahim, R. W. P. R; Mocarzel, R. C. S. (2021) A Federalização da Luta Marajoara. (In) Mocarzel, R. C. S. (Org.). Lutas/Artes Marciais/Esportes de Combate em Educação Física. Curitiba: Apris, p. 49-51

Pacheco, A. S. A conquista do Ocidente Marajoara: índios, portugueses e religiosos em reivindicações históricas. (2010). In: Schaan, D. P.; Martins, C. P. (Orgs). Muito além dos campos: arqueologia e história da Amazônia Marajoara. Belém, PA: GKNORONHA.

Rodrigues, A. I. C., \& Antunes, M. M. (2019). Ensinando lutas na escola: percepções e expectativas de dirigentes do ensino fundamental. Revista Valore, 4(1), 885-899.

Rosário, L. F. R., \& Darido, S. C. (2005). A sistematização dos conteúdos da educação física na escola: a perspectiva dos professores experientes. Motriz. 
Research, Society and Development, v. 11, n. 3, e23311326454, 2022

(CC BY 4.0) | ISSN 2525-3409 | DOI: http://dx.doi.org/10.33448/rsd-v11i3.26454

Journal of Physical Education. UNESP, 167-178.

Rufino, L. G. B., \& Darido, S. C. (2012). Pedagogia do esporte e das lutas: em busca de aproximações. Revista brasileira de educação física e esporte, 26, 283-300.

Santos, C. A. F., \& de Freitas, R. G. (2018). Luta marajoara e memória: práticas" esquecidas" na educação física escolar em Soure-Marajó. Caderno de Educação Física e Esporte, 16(1), 57-67.

Seabra, J. P., Campos, I. S. L., \& Antunes, M. M. (2020). Luta marajoara: uma perspectiva a partir do discurso de atletas. Revista Valore, 5, 5024.

Zabala, A. (1998). A prática educativa: como ensinar. Artmed. 